\title{
Sex-Based Differences in the Presentation of Myocardial Infarction
}

\author{
Jeremy Mayfield ${ }^{1,2}$, Muneet Gill ${ }^{3}$, Helen J. Zhang ${ }^{3}$, Latha Ganti 4, 5, 2, 1 \\ 1. Emergency Medicine, HCA Healthcare Graduate Medical Education Consortium Emergency Medicine Residency \\ Program of Greater Orlando, Orlando, USA 2. Emergency Medicine, Osceola Regional Medical Center, Kissimmee, USA \\ 3. Emergency Medicine, Brown University, Providence, USA 4. Emergency Medicine, Envision Physician Services, \\ Plantation, USA 5. Emergency Medicine, University of Central Florida College of Medicine, Orlando, USA
}

Corresponding author: Latha Ganti, latha.ganti@ucf.edu

\begin{abstract}
The authors report a case of a middle-aged female presenting with a chief complaint of shoulder pain. Workup revealed a non-ST-segment elevation myocardial infarction. The typical symptoms of myocardial infarction and the evaluation of a non-ST-segment elevation myocardial infarction are discussed. The authors highlight the sex-based differences in the presentation of myocardial infarction and remind us to keep a broad differential and consider atypical presentations.
\end{abstract}

Categories: Cardiology, Emergency Medicine

Keywords: non st-segment elevation myocardial infarction, sex-based differnces in coronary disease, sex-based differences in acute coronary syndrome, chest pain, emergency cardiology

\section{Introduction}

Myocardial infarction is defined as a decrease in or absence of blood flow to any area of the heart, resulting in damage to the muscle. This occurs because the formation of plaques in the interior walls of the arteries reduces blood flow to the heart, depriving it of oxygen [1]. Myocardial infarction classically presents as a deep, visceral chest pain, which is typically described as heavy, squeezing, tightness, crushing, and sometimes stabbing or burning pain. The pain may radiate to corresponding dermatomes such as the epigastrium, shoulders, arms, back (interscapular region), lower jaw, and neck [2]. Some of the feared complications of myocardial infarction include mechanical, arrhythmic, ischemic, and inflammatory (early pericarditis and post-myocardial infarction syndrome) sequelae, as well as left ventricular mural thrombus [3]. Moreover, approximately $5 \%$ of patients with myocardial infarction will suffer cardiogenic shock, which has a mortality of $\geqslant 30 \%[4]$. Thus, a timely diagnosis is imperative.

The typical presentation discussed above of myocardial infarction is often not present in women, as this case highlights. Thus, it is important to take this discrepancy into consideration with female patients. The authors discuss their patients in the context of sex-based differences in presentation for myocardial infarction.

Review began 07/27/2021 Review ended 07/28/2021 Published 08/05/2021

๑) Copyright 2021

Mayfield et al. This is an open access article distributed under the terms of the Creative Commons Attribution License CC-BY 4.0., which permits unrestricted use, distribution, and reproduction in any medium, provided the original author and source are credited.

\section{Case Presentation}

A 63-year-old female with a 60-pack-year smoking history presented to the emergency department with left shoulder pain. The shoulder pain started two weeks prior after she was lifting a box. The pain was located on the anterior portion of her left shoulder. The area was exquisitely tender to palpitation, and she had limited range of motion of her left shoulder due to the pain. The patient also endorsed one episode of vomiting that occurred one day prior when the pain was at its maximal intensity 10/10 and spread to her left chest area. She rated her pain a 5/10 at rest. She described the pain as tight. On assessment, the patient was sitting comfortably in the bed. Vital signs were: blood pressure in the right arm 146/98 mmHg, left arm 144/96 $\mathrm{mmHg}$, heart rate 117 beats per minute, pulse oximetry $100 \%$ on room air, respiratory rate 18 breaths per minute, and temperature $36.9^{\circ} \mathrm{C}$. Laboratory analysis was unremarkable (Table 1). 


\section{Cureus}

\begin{tabular}{|c|c|c|}
\hline & Reference Ranges & Laboratory Analysis \\
\hline Sodium (mmol/L) & $136-145$ & 132 \\
\hline Potassium (mmol/L) & $3.7-5.1$ & 3.9 \\
\hline Chloride (mmol/L) & $98-107$ & 105 \\
\hline Carbon dioxide (mmol/L) & $21-32$ & 21 \\
\hline Blood Urea Nitrogen (mg/dL) & 7-18 & 10 \\
\hline Creatinine (mg/dL) & $0.55-1.3$ & 0.78 \\
\hline Glucose (mg/dL) & 74-106 & 325 \\
\hline Calcium (mg/dL) & 8.4-19.1 & 8.9 \\
\hline Troponin I (ng/mL) & Equal/less than 0.05 & 4.82 \\
\hline White Blood Cell count $\left(10^{3} / \mu \mathrm{L}\right)$ & $4.0-10.5$ & 22.7 \\
\hline Red Blood Cell count $\left(10^{6} / \mu \mathrm{L}\right)$ & $3.93-5.22$ & 4.59 \\
\hline Hemoglobin (g/dL) & 13.7-17.5 & 14 \\
\hline Hematocrit (\%) & $34.1-44.9$ & 43.4 \\
\hline Platelet Count $\left(10^{3} / \mu \mathrm{L}\right)$ & $150-450$ & 276 \\
\hline Immature Gran (\%) & $0.0-0.4$ & 0.5 \\
\hline Neutrophils (\%) & $34.0-71.1$ & 89.4 \\
\hline Lymphocytes (\%) & 19.3-51.7 & 7.6 \\
\hline Monocytes (\%) & $4.7-12.5$ & 2.4 \\
\hline Eosinophils (\%) & $0.7-5.8$ & 0 \\
\hline Basophils (\%) & $0.1-1.2$ & 0.1 \\
\hline
\end{tabular}

TABLE 1: Patient's initial laboratory results

The electrocardiogram however demonstrated ST elevation in V1-V2 and ST depression in II, III, and aVF (Figure 1). 


\section{Cureus}

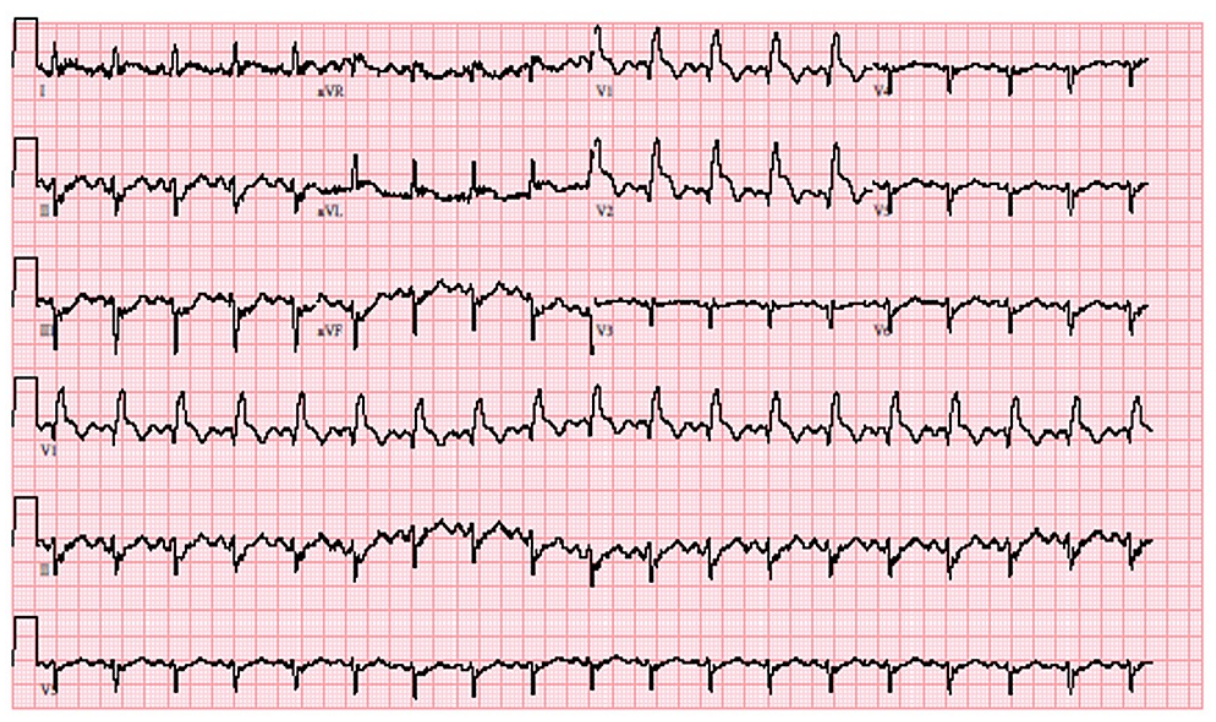

FIGURE 1: Patient's electrocardiogram demonstrating ST elevation

The patient had never had symptoms like this before. She denied drug use including cocaine or marijuana. She was otherwise healthy, denied fever, chills, cough, diarrhea, hematuria, dysuria, or recent travels. Her only allergy was to fluoroquinolones.

The patient's symptoms were not typical for other emergent causes of shoulder or chest pain such as aortic dissection, pericarditis, pneumonia, pneumothorax, Boerhaave's syndrome, pneumomediastinum, or shingles. The patient was emergently taken to cardiac catheterization where she was found to have triple vessel disease. Cardiothoracic surgery was consulted, and the patient underwent emergent coronary artery bypass grafting $(\mathrm{CABG})$ due to continued chest pain. Nine days later the patient was discharged home.

\section{Discussion}

This case demonstrates an unusual presentation of myocardial infarction and illustrates the importance of sex-based criteria when it comes to symptoms and diagnosis. Commonly recognized signs and symptoms of myocardial infarction include chest pain, heart palpitations, and radiating arm pain [1]. However, women are far less likely to have chest pain as the primary symptom and instead can present with malaise, nausea, and extremity discomfort [5]. A separate meta-analysis also demonstrated that women are more likely to present with shoulder pain, emesis, and shortness of breath, two of which were observed in our patient [6]. Sexbased differences also exist in electrocardiography, with women presenting with less ST-segment elevation in their electrocardiograms (Figure 2) [7]. 


\section{Cureus}

\section{Sex-Based Differences in Symptoms for Myocardial Infarction}

805,000 Americans will have a heart attack each year.

Although men are more likely to experience a heart attack at a younger age, women have much lower survival rates. Gender disparities embedded in the healthcare field are to blame for these statistics.

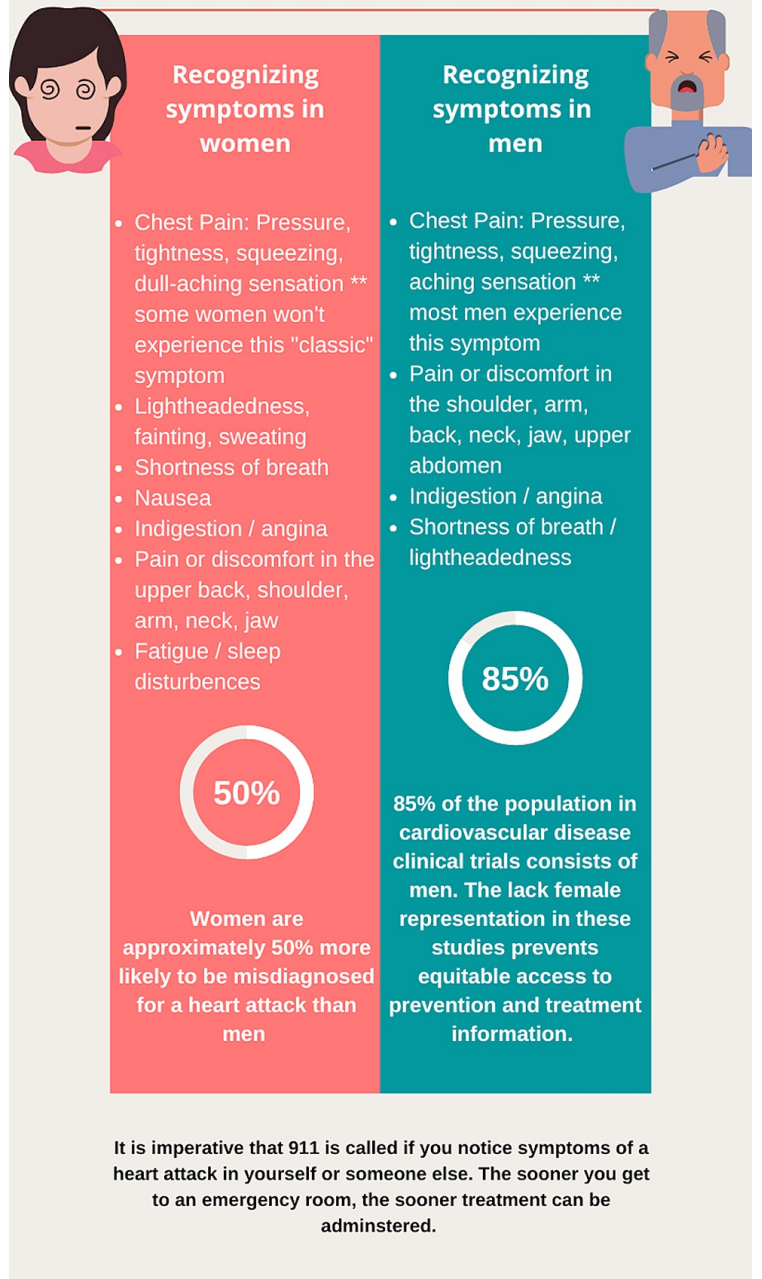

\section{FIGURE 2: Sex-based differences in symptom presentation for myocardial infarction}

More importantly, women also have a higher rate of missed diagnoses; in a study of 563 individuals, approximately $30 \%$ of myocardial infarctions were undiagnosed in women compared to $16 \%$ in men [8]. This lack of recognition was mainly attributed to less specific cardiac symptoms that were self-reported by the patients. Unrecognized myocardial infarction patients also had higher glucose levels, hypertension, and positive smoking status, which are now used as increased predictors of myocardial infarction in women compared to men. This previous misclassification questions the accuracy of past literature reviews, awareness campaigns, and treatment courses that are typically used in the ED.

In recent years, there has been increasing pressure to characterize the sex-based differences for the initial presentation of myocardial infarction. For example, the description of chest pain is vastly different for men and women. Women more often define their pain as "tight" or a "dull ache," whereas men describe their pain 
as “stabbing” [5]. Women also often describe a cluster of symptoms compared to a “classic angina," meaning that their symptoms may often be mistaken for other illnesses because of a varied presentation [9]. Furthermore, smoking and diabetes are more sensitive risk factors of about two-fold in women compared to men.

Troponin assays are also extremely important in diagnosing myocardial injury and infarction. High sensitivity cardiac troponin assays can more intricately assess variation between sexes, with women having a significantly lower end limit of $16 \mathrm{ng} / \mathrm{L}$ compared to $34 \mathrm{ng} / \mathrm{L}$ in men in order to establish a decision threshold [7].

\section{Conclusions}

It has been shown that defining sex-specific criteria is highly useful in distinguishing myocardial infarction from other potential diagnoses. This case illustrates these differences as seen in the patient's primary symptom of shoulder pain, clustering symptoms of emesis and nausea, and the description of the chest pain. Given the significant differences in symptomatology and lab testing between sexes, it is important for clinicians to consider sex and gender disparities for more efficient diagnosis and treatment.

\section{Additional Information}

\section{Disclosures}

Human subjects: Consent was obtained or waived by all participants in this study. HCA Centralized Algorithms for Research Rules on IRB Exemptions (CARRIE) issued approval 2021-519. HCA Centralized Algorithms for Research Rules on IRB Exemptions (CARRIE)/IRB manager issued approval 2021-519. Based on the information provided and attested as true, the research plan described does not require IRB oversight. This is because you are either a) not engaging in research with human subjects as defined by federal regulations; b) engaging in research with human subjects deemed excluded from IRB oversight per 45CFR46.102(1); or c) engaging in research with sufficient human subject protections in the design to meet one or more IRB exemption criteria set forth in 45CFR46.104. Conflicts of interest: In compliance with the ICMJE uniform disclosure form, all authors declare the following: Payment/services info: All authors have declared that no financial support was received from any organization for the submitted work. Financial relationships: All authors have declared that they have no financial relationships at present or within the previous three years with any organizations that might have an interest in the submitted work. Other relationships: All authors have declared that there are no other relationships or activities that could appear to have influenced the submitted work.

\section{References}

1. Lu L, Liu M, Sun R, Zheng Y, Zhang P: Myocardial infarction: symptoms and treatments. Cell Biochem Biophys. 2015, 72:865-7. 10.1007/s12013-015-0553-4

2. Boateng S, Sanborn T: Acute myocardial infarction. Dis Mon. 2013, 59:83-96. 10.1016/j.disamonth.2012.12.004

3. Mullasari AS, Balaji P, Khando T: Managing complications in acute myocardial infarction. J Assoc Physicians India. 2011, 59 Suppl:43-8.

4. Chapman AR, Adamson PD, Shah AS, et al.: High-sensitivity cardiac troponin and the universal definition of myocardial infarction. Circulation. 2020, 141:161-71. 10.1161/CIRCULATIONAHA.119.042960

5. Nuding S, Werdan K, Prondzinsky R: Optimal course of treatment in acute cardiogenic shock complicating myocardial infarction. Expert Rev Cardiovasc Ther. 2018, 16:99-112. 10.1080/14779072.2018.1425141

6. Ferry AV, Anand A, Strachan FE, et al.: Presenting symptoms in men and women diagnosed with myocardial infarction using sex-specific criteria. J Am Heart Assoc. 2019, 8:e012307. 10.1161/JAHA.119.012307

7. Mauvais-Jarvis F, Merz NB, Barnes PJ, et al.: Sex and gender: modifiers of health, disease, and medicine Lancet. 2020, 396:565-82. 10.1016/S0140-6736(20)31561-0

8. van der Ende MY, Juarez-Orozco LE, Waardenburg I, et al.: Sex-based differences in unrecognized myocardial infarction. J Am Heart Assoc. 2020, 9:e015519. 10.1161/JAHA.119.015519

9. McGregor AJ, Beauchamp GA, Wira CR 3rd, Perman SM, Safdar B: Sex as a biological variable in emergency medicine research and clinical practice: a brief narrative review. West J Emerg Med. 2017, 18:1079-90. 10.5811/westjem.2017.8.34997 